\title{
Strong gravitational lensing: relativity in action
}

\author{
Joachim Wambsganss ${ }^{1,2}$ \\ ${ }^{1}$ Astronomisches Rechen-Institut, Zentrum für Astronomie der Universität Heidelberg, \\ Mönchhofstr. 12-14, 69120 Heidelberg, Germany \\ email: jkw@uni-hd.de \\ ${ }^{2}$ Bohdan Paczynski Visitor, Dept. of Astrophysical Sciences, Princeton University, \\ Princeton, NJ 08540, USA
}

\begin{abstract}
Deflection of light by gravity was predicted by Einstein's Theory of General Relativity and observationally confirmed in 1919. In the following decades, various aspects of the gravitational lens effect were explored theoretically, among them measuring the Hubble constant from multiple images of a background source, making use of the magnifying effect as a gravitational telescope, or the possibility of a "relativistic eclipse" as a perfect test of GR. Only in 1979, gravitational lensing became an observational science when the first doubly imaged quasar was discovered. Today lensing is a booming part of astrophysics and cosmology. A whole suite of strong lensing phenomena have been investigated: multiple quasars, giant luminous arcs, Einstein rings, quasar microlensing, and galactic microlensing. The most recent lensing application is the detection of extrasolar planets. Lensing has contributed significant new results in areas as different as the cosmological distance scale, mass determination of galaxy clusters, physics of quasars, searches for dark matter in galaxy halos, structure of the Milky Way, stellar atmospheres and exoplanets. A guided tour through some of these applications will illustrate how gravitational lensing has established itself as a very useful universal astrophysical tool.
\end{abstract}

Keywords. light deflection, gravitational lensing, cosmology, extrasolar planets

\section{The History of Strong Lensing}

Gravitational lensing is considered a relatively new field in astrophysics. However, the history of light deflection is more than 200 years old (see in more detail in Wambsganss [1998] or in Schneider, Kochanek \& Wambsganss [2006]). As early as 1784, Michell considered the deflection of light by the gravity of other bodies. In 1801, Soldner published a paper on light deflection, in which he determined - based on Newtonian mechanics the deflection of a light ray just passing the solar limb to

$$
\alpha_{\odot, \text { Soldner }}=\frac{2 G M_{\odot}}{c^{2} R_{\odot}}=0.87 \operatorname{arcsec}
$$

(with $G$ - gravitational constant, $c$ - velocity of light, $M_{\odot}$ - mass of the sun, $R_{\odot}$ - radius of the sun). More than 100 years later, Einstein worked on the same problem and derived the same value (Einstein 1911). In fact, an expedition headed by Erwin Freundlich from Potsdam was set up to test this prediction during the solar eclipse in September 1914 on the Crimean Peninsula. However, another eclipse had started to darken Europe, World War I had begun and the scientists plus their equipment were captured by Russian soldiers. No harm was done to either scientists or equipment, they were later released and sent home, but the solar eclipse had passed and the measurement could not be made. Only after the General Theory of Relativity was finished, Einstein published the value 
of

$$
\alpha_{\odot, \text { Einstein } 1915}=\frac{4 G M_{\odot}}{c^{2} R_{\odot}}=1.74 \text { arcsec, }
$$

for the light deflection at the solar limb, which was measured and proven to be correct in the famous solar eclipse expeditions led by Eddington in 1919 (Dyson et al. 1920).

In the 1920s/1930s, there were a few papers dealing with lensing, e.g., Chwolson investigated the situation of double imaging. In particular he figured out that for perfect alignment between lens and source the result would be a ring-like image (Chwolson 1924). Einstein looked again into this issue and derived the magnifications for the double images of a background star lensed by an intervening foreground star, but he was very sceptical about the possibility of observing this gravitational lensing effect (Einstein 1936). Henry Norris Russell had obtained Einstein's 1936 paper as a preprint. It impressed him quite a bit, so he immediately wrote a column for Scientific American entitled "A Relativistic Eclipse" (Russell 1937), in which he emphasized that the "Einstein effect" provides a perfect (but unavailable) test for General Relativity, and that the effects would be "conspicuous to the immediate gaze". He described the consequences of an eclipse in the Sirius A and B system, imagining an observer sitting on a planet around the white dwarf Sirius B, at a distance such that the angular size of Sirius B just matches the angular size of Sirius A. He illustrates the image shapes and describes what we call "arcs" today as "bright crescents" and images have "developed pointed horns". Fritz Zwicky immediately applied Einstein's idea on galaxies, he was convinced that "nebulae" should and would act as gravitational lenses, for him this appeared to be an unavoidable consequence (Zwicky 1937a,b) of the light deflection theory. Zwicky recognized the potential of applying gravitational lensing to extragalactic nebulae: he proposed that this would offer an additional test for general relativity, that the gravitational lens effect could be used to study fainter objects (effectively increasing the aperture of our telescopes), he emphasized that lensing would provide a powerful method to measure the masses of the "nebulae" (i.e. dark matter!), and he proposed that splittings up to 30 arcsecond should be expected.

In the 1960s there was another wave of theoretical investigations of the lensing effect. In particular, Refsdal showed that one can determine the Hubble constant from the time delay between the images of a multiply lensed quasar (Refsdal 1964). And finally in 1979, Dennis Walsh and colleagues discovered the first doubly imaged quasar Q0957+561 (Walsh et al. 1979). Although the deflection of light at the solar limb - hailed as the first experiment to confirm a prediction of Einstein's theory of General Relativity happened already in 1919, it took more than half a century to establish this phenomenon observationally in some other environment.

\section{The Basics of Strong Lensing}

The path, the size and the cross section of a light bundle propagating through spacetime, in principle, are affected by all the matter between the light source and the observer. For most practical purposes one can assume that the lensing action is dominated by a single matter inhomogeneity at some location between source and observer. This is usually called the "thin lens approximation": all the action of deflection is thought to take place at a single distance. Here the basics of lensing will be briefly derived and explained in the thin lens approxmation: lens equation, Einstein radius, image positions and 
magnifications, time delay. More detailed reviews/introductions on lensing can be found in, e.g., Schneider et al. (2006).

\subsection{Lens Equation}

The basic setup for such a simplified gravitational lens scenario involving a point source and a point lens is displayed in Figure 1. The three ingredients in such a lensing situation are the source $\mathrm{S}$, the lens $\mathrm{L}$, and the observer O. Light rays emitted from the source are deflected by the lens. For a point-like lens, there will always be (at least) two images $\mathrm{S}_{1}$ and $\mathrm{S}_{2}$ of the source. With external shear - due to the tidal field of objects outside but near the light bundles - there can be more images. The observer sees the images in directions corresponding to the tangents to the real incoming light paths.

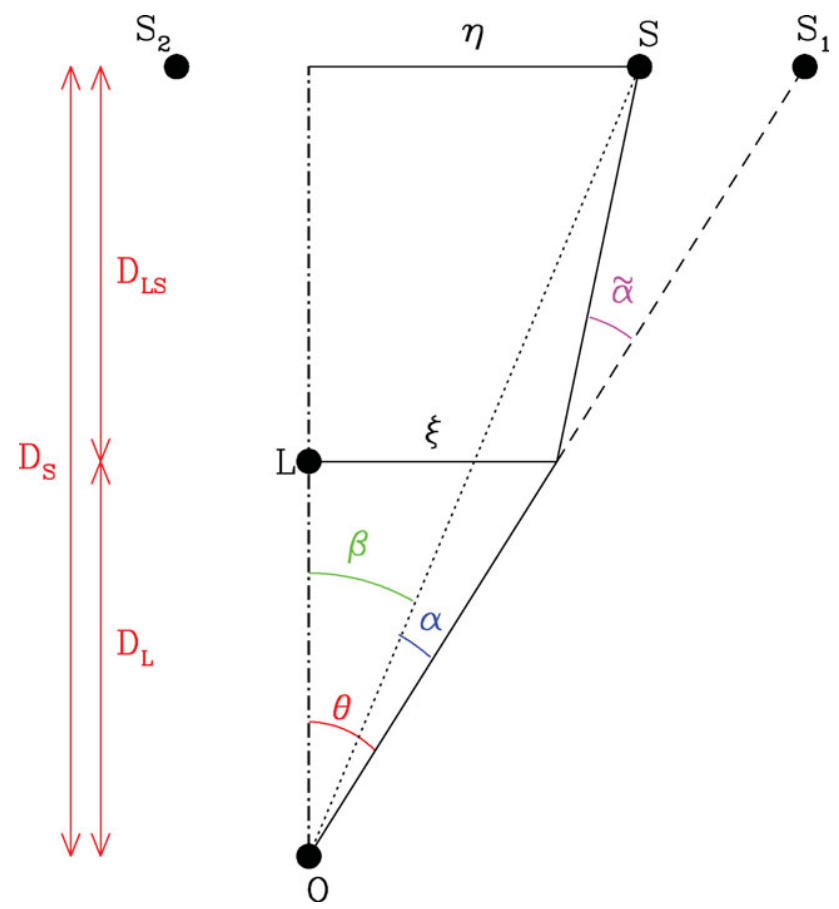

Figure 1. The relations between the various angles and distances involved in the lensing setup can be derived for $\tilde{\alpha} \ll 1$ and formulated in eq.(2.3), the lens equation. The symbols ' $\mathrm{O}$ ', ' $\mathrm{L}$ ', and ' $\mathrm{S}$ ' mean 'observer', 'lens', and 'source', respectively. ' $\mathrm{S}_{1}$ ' and ' $\mathrm{S}_{2}$ ' are the two apparent positions of the doubly imaged source. The angular diameter distances $\mathrm{D}_{L}, \mathrm{D}_{S}$, and $\mathrm{D}_{L S}$ are between observer-lens, observer-source, and source-lens. All angles involved are small compared to one.

In Figure 1, the corresponding angles and angular diameter distances $D_{L}, D_{S}, D_{L S}$ are indicated. In the thin-lens approximation the hyperbolic paths are approximated by their asymptotes. In the circular-symmetric case the deflection angle is given as

$$
\tilde{\alpha}(\xi)=\frac{4 G M(\xi)}{c^{2}} \frac{1}{\xi},
$$

where $M(\xi)$ is the mass of the lens inside a radius $\xi$. In this depiction the origin is chosen at the observer. From the diagram it can be seen that the following relation holds:

$$
\theta D_{S}=\beta D_{S}+\tilde{\alpha} D_{L S}
$$


(for $\theta, \beta, \tilde{\alpha} \ll 1$; this condition is fulfilled in practically all astrophysically relevant situations). With the definition of the reduced deflection angle as $\alpha(\theta)=\left(D_{L S} / D_{S}\right) \tilde{\alpha}(\theta)$, this lens equation can be expressed as:

$$
\beta=\theta-\alpha(\theta) .
$$

For real situations, all the astrophysics is "hidden" in the deflection angle $\alpha(\theta)$, which can become a quite complicated function for many lensing objects or an extended mass distributions.

\subsection{Einstein Radius}

For a point lens of mass $M$ the deflection angle is given by equation (2.1). Plugging it into equation (2.3) and using the relation $\xi=D_{L} \theta$ (cf. Figure 1) one obtains:

$$
\beta(\theta)=\theta-\frac{D_{L S}}{D_{L} D_{S}} \frac{4 G M}{c^{2} \theta} .
$$

For the special case in which the source lies exactly behind the lens $(\beta=0)$, due to the symmetry a ring-like image occurs whose angular radius is called Einstein radius $\theta_{E}$ :

$$
\theta_{E}=\sqrt{\frac{4 G M}{c^{2}} \frac{D_{L S}}{D_{L} D_{S}}} .
$$

The Einstein radius defines the angular scale for a lens situation. For a massive galaxy with a mass of $M=10^{12} M_{\odot}$ at a redshift of $z_{L}=0.5$ and a source at redshift $z_{S}=2.0$ (here $H=50 \mathrm{~km} \mathrm{sec}^{-1} \mathrm{Mpc}^{-1}$ is used as the value of the Hubble constant and an Einstein-deSitter universe) the Einstein radius is

$$
\theta_{E} \approx 1.8 \sqrt{\frac{M}{10^{12} M_{\odot}}} \operatorname{arcsec}
$$

(note that for cosmological distances in general $D_{L S} \neq D_{S}-D_{L}$ !). For a galactic microlensing scenario in which stars in the disk of the Milky Way act as lenses for bulge stars close to the center of the Milky Way, the scale defined by the Einstein radius is

$$
\theta_{E} \approx 0.5 \sqrt{\frac{M}{M_{\odot}}} \text { milliarcsec. }
$$

It is obvious from these values that lensing by galaxies can be resolved by normal optical telescopes, whereas the angular scale for lensing by stars is much smaller than the resolution of even the best optical telescopes.

\subsection{Image Positions and Magnifications}

The lens equation (2.3) can be re-formulated in the case of a single point lens:

$$
\beta=\theta-\frac{\theta_{E}^{2}}{\theta} .
$$

Solving this for the image positions $\theta$, one finds that an isolated point source always produces two images of a background source. The positions of the images are given by the two solutions:

$$
\theta_{1,2}=\frac{1}{2}\left(\beta \pm \sqrt{\beta^{2}+4 \theta_{E}^{2}}\right)
$$


The magnification of an image is defined by the ratio between the solid angles of the image and the source, since the surface brightness is conserved. Hence the magnification $\mu$ is given as

$$
\mu=\frac{\theta}{\beta} \frac{d \theta}{d \beta} .
$$

In the symmetric case above the image magnification can be written as (by using the lens equation):

$$
\mu_{1,2}=\left(1-\left[\frac{\theta_{E}}{\theta_{1,2}}\right]^{4}\right)^{-1}=\frac{u^{2}+2}{2 u \sqrt{u^{2}+4}} \pm \frac{1}{2}
$$

Here $u$ is defined as the "impact parameter", the angular separation between lens and source in units of the Einstein radius: $u=\beta / \theta_{E}$. The magnification of one image (the one inside the Einstein radius) is formally negative. This means it has negative parity: it is mirror-inverted. For $\beta \rightarrow 0$ the magnification diverges: in the limit of geometrical optics the Einstein ring of a point source has infinite magnification $\dagger$ ! The sum of the absolute values of the two image magnifications is the total magnification $\mu$ :

$$
\mu=\left|\mu_{1}\right|+\left|\mu_{2}\right|=\frac{u^{2}+2}{u \sqrt{u^{2}+4}} .
$$

Note that this value is (always) larger than onef!

\subsection{Time delay and Fermat's Theorem}

The deflection angle is the gradient of an effective lensing potential $\psi$ (see Schneider 1985). Hence the lens equation can be rewritten as

$$
(\boldsymbol{\theta}-\boldsymbol{\beta})-\nabla_{\theta} \psi=0
$$

or

$$
\nabla_{\theta}\left(\frac{1}{2}(\boldsymbol{\theta}-\boldsymbol{\beta})^{2}-\psi\right)=0
$$

The term in brackets appears as well in the physical time delay function for gravitationally lensed images:

$$
\tau(\boldsymbol{\theta}, \boldsymbol{\beta})=\tau_{\text {geom }}+\tau_{\text {grav }}=\frac{1+z_{L}}{c} \frac{D_{L} D_{S}}{D_{L S}}\left(\frac{1}{2}(\boldsymbol{\theta}-\boldsymbol{\beta})^{2}-\psi(\theta)\right) .
$$

This time delay surface is a function of the image geometry $(\boldsymbol{\theta}, \boldsymbol{\beta})$, the gravitational potential $\psi$, and the distances $D_{L}, D_{S}$, and $D_{L S}$. The first part - the geometrical time delay $\tau_{\text {geom }}$ - reflects the extra path length compared to the direct line between observer and source. The second part - the gravitational time delay $\tau_{\text {grav }}$ - is the retardation due to the gravitational potential of the lensing mass (known and confirmed as Shapiro delay in the solar system). From equations (2.14) and (2.15) it follows that the gravitationally lensed images appear at locations that correspond to extrema in the light travel time, which reflects Fermat's principle in gravitational-lensing optics.

The (angular-diameter) distances that appear in equation (2.15) depend on the value of the Hubble constant (Weinberg 1972); therefore, it is possible to determine the latter

$\dagger$ Due to the fact that physical objects have a finite size, and also because at some limit wave optics has to be applied, in reality the magnification stays finite.

$\ddagger$ This does not violate energy conservation, since this is the magnification relative to an "empty" universe and not relative to a "smoothed out" universe. 
by measuring the time delay between different images and using a good model for the effective gravitational potential $\psi$ of the lens.

\subsection{The Effects of strong lensing}

The effects of strong lensing can be summarized in four aspects:

- change of position: This effect was used as the first confirmation of General Relativity in 1919 with the offset of stellar positions close to the solar limb (Dyson et al. 1920). However, in general this is normally not observable, because one needs a "beforeand-after"-scenario in order to compare two angular positions. There is some hope that in the future astrometric microlensing can help measure this effect (Treyer \& Wambsganss 2004).

- distortion: Extended sources that are affected by lensing are distorted; to first order, circular sources are deformed into arclets, arcs or even Einstein rings.

- (de)magnification: Typically only the magnification effect of lensing is considered. In stellar microlensing a few events have been measured with magnifications as large as a factor 1000. However, since lensing does not create any photons, in order to compensate for this, some sources will appear fainter than without lensing. The distribution, however, is very skewed: almost all images appear slightly demagnified, whereas a small fraction of objects are highly magnified. This means, that in fact there are no standard candles in the universe (Wambsganss et al. 1997).

- multiple images: Multiple images of course are the most dramatic effect of lensing. So far, we know of more than 100 multiple quasar systems, and even more galaxy clusters that produce giant luminous arcs.

In Figure 2 all these effects are visualized assuming a particular source profile with some internal structure.
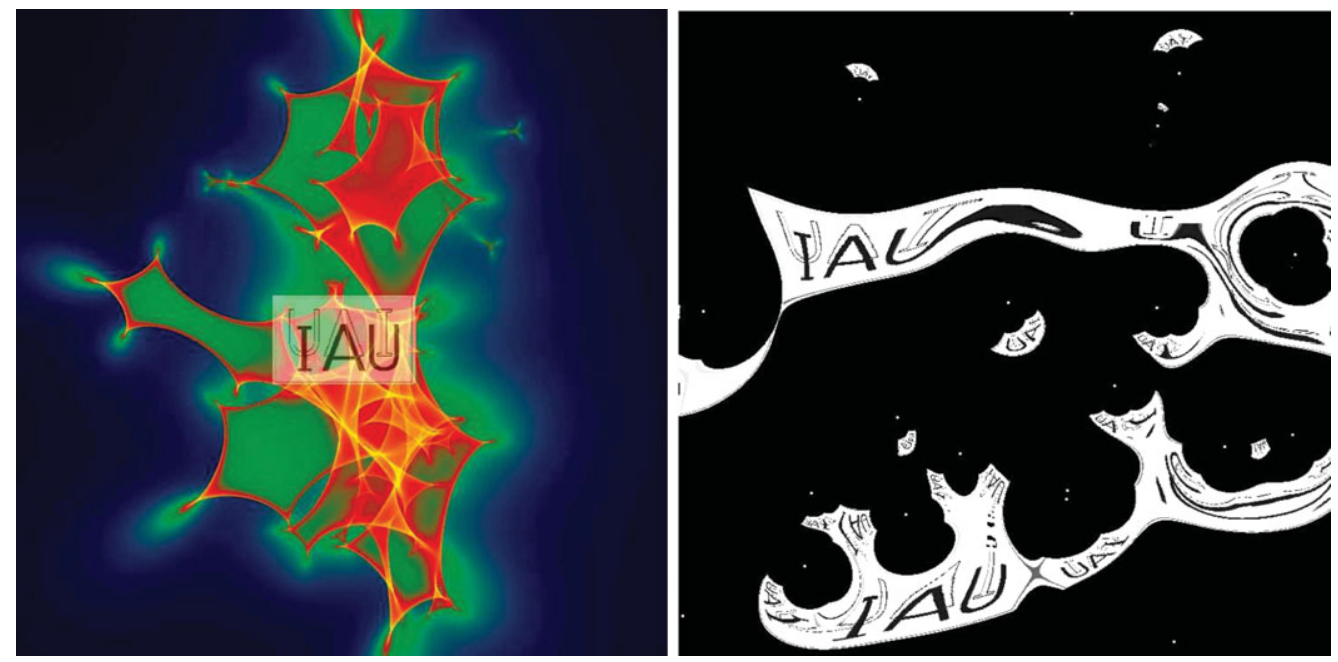

Figure 2. Magnification pattern produced by the stars in a lensing galaxy, with a particular source profile superposed (left); resulting configuration with distorted, offset, magnified multiple images (right).

\subsection{How can we observe strong lensing phenomena?}

Depending on the mass scale of the lens, there are two very different ways to observe strong lensing: 
- "Statically" (images): For lensing objects with masses $m \geqslant 10^{8} M_{\odot}$, the angular Einstein radius gets comparable to the resolution of the telescope. This means the lensing effect can be detected by resolving the multiple images and/or the shapes of the distorted images. This regime is occasionally called macro-lensing.

- "Dynamically" (brightness, positions): For lensing objects with masses roughly comparable to a solar mass, the angular Einstein radius is much smaller than the resolution of the telescope. However, due to the relative change between the (angular) positions of lens and source, the magnification (and also the center of light) can change with time. The time scale of these changes (Einstein radius divided by transverse velocity) turns out to be of order years/months/weeks and, hence, the phenomenon is detectable by measuring the apparent brightness frequently over time (in the future, we hope to be able also to measure the positional change of the center-of-light). This regime is called micro-lensing.

\section{The Usefulness of Strong Lensing}

In this section, three examples are presented in which strong lensing is being applied: Galaxy clusters as lenses providing a record time delay and arc statistics as a cosmological tool, galaxies as lenses to be used as microscopes for quasar accretion disks, and finally stars and planets as lenses in the Milky Way.

\subsection{Galaxy Clusters as Lenses: Time Delays and Arc Statistics}

Gravitational lensing directly measures mass density fluctuations along the lines of sight to very distant objects. No assumptions need to be made concerning bias, the ratio of fluctuations in galaxy density to mass density. Hence, strong lensing is a very useful tool to study the statistics of giant luminous arcs. This was done, e.g., regarding the frequency of giant luminous arcs predicted by various cosmological models. In Bartelmann et al. (1998) it was stated that a Lambda-dominated flat cosmological model $\left(\Omega_{\text {matter }}=0.3, \Omega_{\Lambda}=0.7\right.$ known as "concordance cosmology") would underpredict luminous arcs by about an order of magnitude. Later, Wambsganss et al. (2004) showed that this result was based on the assumption that the sources are all at redshift unity. The probability for arcs, however, is a steep function of source redshift. If one allows for sources at redshift two or three (some of the observed arcs are even at higher redshifts), then the discrepancy disappears. These results were based on cosmological parameters derived from the WMAP-1 data. More recently, WMAP-3 results published lower values of the normalization, $\sigma_{8}$, which reduces the predicted number of arcs by a factor of 8 or 10 (Wambsganss et al., in preparation). The most recent values for the cosmological parameters based on WMAP-5 results (in particular the slight increase in the normalization) lead to an increase in the frequency of arcs again. Since all three values of $\sigma_{8}$ published by the WMAP mission agree within each other at a level of about $2.5 \sigma$, there is no significant discrepancy between the predicted and measured arc frequencies. The models mainly show the strong dependence on the normalization of the cosmology. If better statistics can be obtained from a larger number of observed arcs, this could be used in turn to predict an interval of $\sigma_{8}$ which is consistent with these observations.

A second example for the usefulness of galaxy clusters as lenses was obtained in the strongly lensing galaxy cluster SDSS J1004+4112, in which a quintuple quasar system was discovered. By monitoring this system over many years, finally two of the three relative time delays could be measured, and a lower limit on the third one could be obtained (see Fohlmeister et al. 2007, 2008): the time delay between images A and B was measured to $\Delta t_{B A}=40.6 \pm 1.8$ days, the wide image pair $\mathrm{C}$ and $\mathrm{A}$ has a relative 
time delay of $\Delta t_{C A}=821.6 \pm 2.1$ days, which is the longest gravitational lens time delay on record so far. For the image pair A and D only a lower limit could be obtained: $\Delta t_{A D}>1250$ days (cf. Figure 3 and for more details the papers by Fohlmeister et al. 2007, 2008).

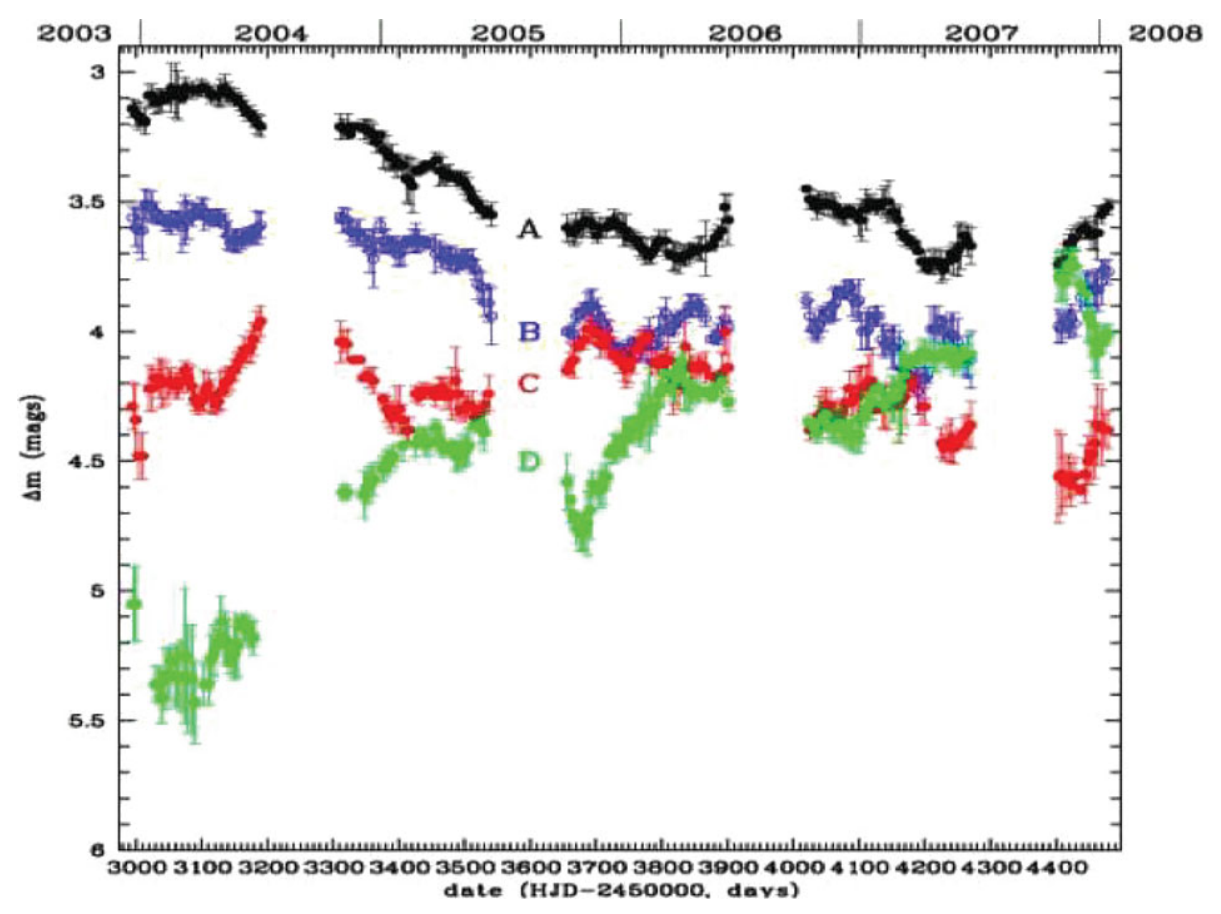

Figure 3. Lightcurves of the four brightest images of the multiple quasar system SDSS J1004+4112 (after Fohlmeister et al. 2008)

\subsection{Galaxies as lenses: Dark Matter and Quasar Accretions Disks}

A number of quasar systems have been explored in detail for microlensing, the effects of stellar mass objects along the line of sight to the quasar images. The most dramatic effects have been seen in the quadruple system Q2237+0305, where four quasar images are centered around the core of the lensing galaxy. Since its discovery, this system has shown uncorrelated fluctuations between the images, which were interpreted as microlensing, see Irwin et al. (1989) and Wambsganss et al. (1990). The problem was, however, the poor coverage in time. Only with the dedicated telescope and the dedicated scientists of the OGLE team, a good time coverage could be reached, with more than 100 data points per year. This observing strategy resulted in a dramatic increase in data quality, see Wozniak et al. (2000). The individual images fluctuate by as much as a factor of two in a few months, and these fluctuations are very well resolved. In this multiple quasar system there is no need to invoke potential dark matter objects as lenses: the four images are seen through the central part of the lensing galaxy, which is full of ordinary main sequence stars. The interpretation of the data is consistent with low-mass stars in the core of the galaxy being the lenses, and the size of the quasar continuum emission region to be of order $10^{15} \mathrm{~cm}$ or smaller (see, e.g., Wambsganss et al. (1990), Wyithe et al. (2000))

Recently, this quasar could be monitored spectro-photometrically over an extended period of time. Eigenbrod et al. (2008) analyzed the data with particular emphasis on 


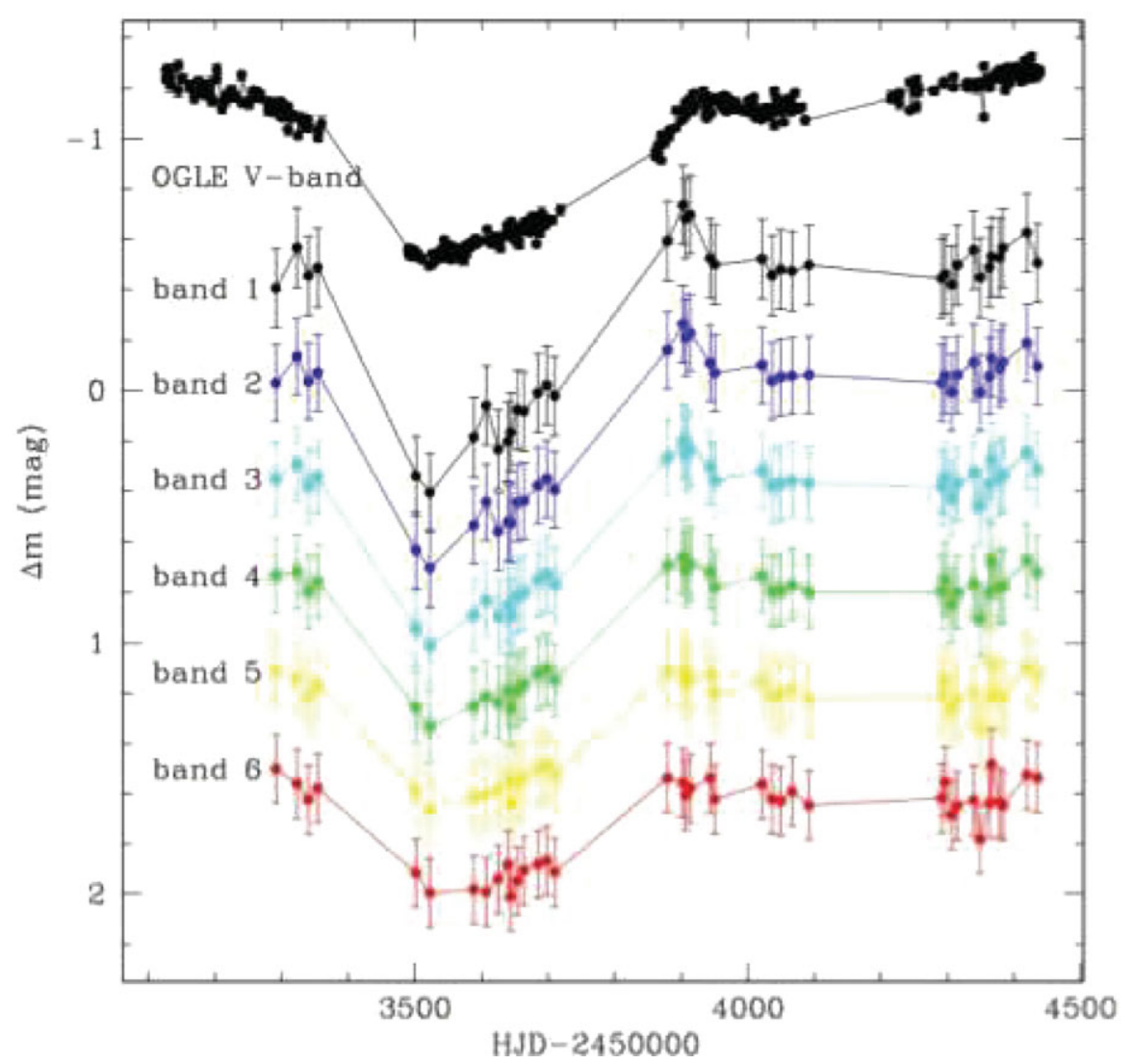

Figure 4. Microlensing lightcurves of the quadruple quasar Q2237+0305 in six bands, clearly indicating the dependence of the microlensing amplitude on the wavelength (from Eigenbrod et al. 2008).

the microlensing amplitudes as a function of wavelength/filter. As can be seen in Figure 4, the amplitudes increase with decreasing wavelength: in the blue wavelength range the microlensing effect is stronger than in the red part. This was originally predicted by Wambsganss \& Paczynski (1991). Here this effect could be analyzed quantitively for the first time. The results show that the accretion disk in the quasar Q2237+0305 is consistent with a standard Shakura-Sunyaev accretion disk, details can be found in Eigenbrod et al. (2008).

\subsection{Stars as Lenses: In Search of extrasolar Planets}

In 1991, it was suggested that a fair fraction of stellar microlensing events towards the Galactic bulge should display signatures of binarity, and that even planetary companions should be detectable (Mao \& Paczynski 1991). Starting in 1993, a number of teams (MACHO, EROS, OGLE, MOA) monitored of order $10^{7}$ stars in the bulge in order to detect microlensing effects of intermediate stars or dark compact objects (Alcock et al. 2000; Laserre et al. 2000; Udalski et al. 1997). By now, more than 3000 microlensing events towards the galactic bulge have been found, currently over 800 events are detected per season. About $10 \%$ of them show the signature of binary lenses. This data set allows, among other things, to study the mass distribution of the Galactic disk with 
unprecedented accuracy. But one of the main goals of these monitoring experiments is still the detection of planets around the lensing stars.

In addition to the groups mentioned above, there are two other teams (PLANET, MicroFUN) who specialized in following up of current stellar lensing events with good photometric accuracy and very high temporal coverage, in order to find possible small deviations from the smooth single-lens lightcurve, which would be the signature of a planet. The signatures of planets are of short duration (of order hours) and typically have small amplitudes (a few percent), as was shown, e.g., in Wambsganss (1997). But the main aspect are: such planetary deviations at stellar microlensing lightcurves are rare: even if all stars had planets, only a small fraction of the microlensing lightcurves would show their signatures, due to the geometric path of the background star with respect to the planetary caustic.

Finally, in April 2004 the first microlensing planet was announced: The MOA/OGLE/ MicroFUN teams announced their detection of a microlensing event which can be explained only with a very low mass companion to the primary star: OGLE 2003-BLG-235 or MOA 2003-BLG-53. The result is published in Bond et al. (2004). In the original words of the authors:

"A short-duration ( $\sim 7$ days) low-amplitude deviation in the light curve due to a single-lens profile was observed in both the MOA and OGLE survey observations. We find that the observed features of the light curve can only be reproduced using a binary microlensing model with an extreme (planetary) mass ratio of $0.0039_{-07}^{+11}$ for the lensing system. If the lens system comprises a main-sequence primary, we infer that the secondary is a planet of about 1.5 Jupiter masses with an orbital radius of $\sim 3$ AU."

By now, seven microlensing planets have been published (Bond et al. 2004, Udalski et al. 2005, Beaulieu et al. 2006, Gould et al. 2006, Gaudi et al. 2008, Bennett et al. 2008, Dong et al. 2009). These first unambiguous microlensing planet detections prove: Microlensing as a planet search technique has stepped out of its infancy. It is a viable method which is complementary to other techniques. Microlensing remains the most promising method for the detection of low-mass planets with ground-based techniques. It has been known all along that microlensing is sensitive to the low planet mass regime, that even Earth-mass objects are within the reach. However, even for microlensing the sensitivity is stronger for higher mass planets. So the fact that about half of the planets detected with microlensing are in the low planet mass regime, Neptune-mass to few Earthmass range, allows to draw a robust conclusion: low mass planets must be abundant (more details see in references above).

\section{The Future of Strong Lensing}

In the 30 years of its existence, strong gravitational lensing has changed from an exotic subfield in astronomy into a very useful astrophysical tool which is applied in a large range of mass scales (20 orders of magnitude: from galaxy clusters to Earth-like planets), distance scales (few Gigaparsec to few kiloparsec, or even microparsec, if we include the Sun ...) and angular scales (arcminutes to microarcseconds). In the coming years, strong lensing will definitely help measure dark matter, resolve quasar and stellar luminosity profiles, and find many exoplanets, possibly Earth-like planets, and potentially even moons around extrasolar planets. So my conclusion is that the future of strong lensing is bright and promising. 


\section{References}

Alcock, C., et al. (The MACHO collaboration), ApJ 542, 281 (2000)

Bartelmann, M. et al. A\&A 330, 1 (1998)

Beaulieu, J. P., Bennett, D. P., Fouque, P., et al., Nature, 439, 437 (2006)

Bennett, D. P. et al., ApJ 684, 663 (2008)

Bond, I. A., Udalski, A., Jaroszynski, M., Rattenbury, N. J., Paczynski, B., et al., ApJ 606, L155 (2004)

Chwolson, O. Astron. Nachr. 221, 329 (1924)

Dong, S. et al., ApJ 698, 1826 (2009)

Dyson, F., Eddington, A., Davidson, C., Mem. Roy. Astr. Soc. 62, 291 (1920)

Eigenbrod, A. et al. Astron. Astrophys. 490, 933 (2008)

Einstein, A., Annalen der Physik 35, 898 (1911)

Einstein, A., Science 84, 506 (1936)

Fohlmeister, J., et al. , ApJ, 662, 62 (2007)

Fohlmeister, J., Kochanek, C. S., Falco, E. E., Morgan, C. W., \& Wambsganss, J., ApJ, 676, $761(2008)$

Gaudi, B. S. et al., Science, 319, 927 (2008)

Gould, A., Udalski A., An J., et al., ApJ, 644, L 37 (2006)

Irwin, M. J., Webster, R. L., Hewett, P. C., Corrigan, R. T., \& Jedrzejewski, R. I., ApJ 98, 1989 (1989)

Lasserre, T., et al. (The EROS collaboration), A\&A 355, L39 (2000)

Mao, S., Paczyński, B. ApJ 374, L37 (1991)

Refsdal, S. MNRAS 128, 307 (1964)

Russel, H. N. Scientific American (February 1937)

Schneider, P., A\&A 143, 413 (1985)

Schneider, P., Kochanek, C. S., \& Wambsganss, J. "Gravitational Lensing: Strong, Weak, Micro" (Saas-Fee Advanced Course 33, Editors G. Meylan, P. Jetzer, P. North (Springer-Verlag, Berlin, 2006)

Treyer, M. \& Wambsganss, J. A\&A, 416, 19 (2004)

Udalski, A., Kubiak, M., \& Szymanski, M. Acta Astron. 47, 319 (1997)

Udalski, A., Jaroszy, Å., ski, M., Paczy, Å., ski, B., et al., ApJ, 628, L109 (2005)

Walsh, D., Carswell, R. F., \& Weymann, R. J., Nature 279, 381 (1979)

Wambsganss, J., Paczyński, B., \& Schneider, P. ApJ 358, L33 (1990)

Wambsganss, J. \& Paczyński, B. AJ 102, 864 (1991)

Wambsganss, J., Bode, P. \& Ostriker, J. P. ApJ 606, L93 (2004)

Wambsganss, J., MNRAS 284, 172 (1997)

Wambsganss, J., Living Reviews in Relativity 1998-12, http://relativity.livingreviews.org/Articles/lrr-1998-12 (1998)

Weinberg, S.: Gravitation and Cosmology (Wiley, New York, 1972)

Wozniak, P. R., Udalski, A., Szymanski, M., et al. ApJ 540, L65 (2000)

Wyithe, J. S. B., Webster, R. L., \& Turner, E. L. MNRAS 318, 762 (2000)

Zwicky, F., Phys. Rev. 51, 290 (1937a)

Zwicky, F., Phys. Rev. 51, 679 (1937b) 